KYUNGPOOK Math. J. 51(2011), 353-364

http://dx.doi.org/10.5666/KMJ.2011.51.4.353

\title{
Duality in an Optimal Harvesting Problem by a Nonlinear Age-Spatial Structured Population Dynamic System
}

\author{
YongKUK KIM* \\ Department of Mathematics, Kyungpook National University, Daegu 702-701, South \\ Korea \\ e-mail : yongkuk@knu.ac.kr \\ Mi Jin LeE AND Il Hyo Jung \\ Department of Mathematics, Pusan National University, Pusan 609-735, South Ko- \\ rea \\ e-mail: jin0624@pusan.ac.kr and ilhjung@pusan.ac.kr
}

ABstract. Duality in the optimal harvesting for a nonlinear age-spatial structured population dynamic model is studied in the framework of optimal control problem. In this paper the duality theory that displays the conjugacy of the primal problem is established and an application is given. Duality theory plays an important role in both optimization theory and methodology and the results may be applied to a realistic biological system on the point of optimal harvesting.

\section{Introduction}

One of the main aim of duality theory is to show that the suprimum (or infimum) for an objective functional of given a control system is equal to the infimun (or suprimum) for the objective functional of its dual system. It is well-known that duality theory is important in both optimization theory and methodology and duality often makes it possible to simplify the computational procedure and to construct a generalized solution of variational problems that not have classical solutions. Existence of an optimal solution to dual problems provides a certificate of optimality for the primal problem. So many authors studied the duality theory for various systems $[3,17,7,15]$. Park and Lee $[12,13]$ derived the duality theory for linear and nonlinear hyperbolic optimal control systems. Bulatov and Krotov [6] dealt with

* Corresponding Author.

Received September 1, 2011; accepted October 4, 2011.

2010 Mathematics Subject Classification: 35K20, 49J20, 49K20.

Key words and phrases: Duality theory, Optimal control problems, Age-structure, Harvesting problem.

This work was carried out with the support of "Cooperative Research Program for Agriculture Science \& Technology Development (Project No. PJ007420)" Rural Development Administration, Republic of Korea. 
the case which Pontrygin maximum principle is inapplicable to the initial problem but it is applicable to the dual problem.

In particular, many authors studied optimal harvesting problems for agedependent population dynamic systems $[2,10,8]$. Fister and Lenhart [9] dealt with a optimal control problem of a competitive population system with age structure. Optimal control problems for age structured systems are of interest for many areas of application, as harvesting, birth control, epidemic disease control $[1,4,5,16]$.

In order to formulate our dual problem on the harvesting problem, we let $\Omega$ be a bounded domain in $\mathbb{R}^{N}$ with a smooth boundary $\partial \Omega, A>0, T>0$. We denote by $u(x, t, a)$ the distribution of individuals of age $a \geq 0$ at time $t \geq 0$ and location $x$ in $\bar{\Omega}$. Let $\beta(x, t, a) \geq 0$ be the natural fertility rate and $\mu(x, t, a, u) \geq 0$ be the natural death rate of individuals of age $a$ at time $t$ and location $x$ and density $u$, where we note that the death rate $\mu$ is related to the density $u$. In fact, until now, most of study for models of age structured population considered the death rate depending on only time and age $[2,5]$. However, in more realistic situation, it may be natural to assume that the death rate also depends on the population density and the location $x$ as well as the time and age.

The model we address is given by the following nonlinear age-spatial structured population dynamic system:

$$
\begin{aligned}
& \frac{\partial u}{\partial t}(x, t, a)+\frac{\partial u}{\partial a}(x, t, a)-k \Delta_{x} u(x, t, a)+\mu(x, t, a, u(x, t, a)) u(x, t, a) \\
& \quad+\Phi\left(P_{w}(x, t)\right) u(x, t, a)=-v(x, t, a) u(x, t, a) \text { in } Q=\Omega \times(0, T) \times(0, A),
\end{aligned}
$$

$$
\begin{gathered}
\frac{\partial u}{\partial \eta}(x, t, a)=0 \text { on } \Sigma=\partial \Omega \times(0, T) \times(0, A), \\
u(x, 0, a)=u_{0}(x, a) \text { in } \Omega \times(0, A), \\
u(x, t, 0)=\int_{0}^{A} \beta(x, t, a) u(x, t, a) d a \text { in } \Omega \times(0, T), \\
P_{w}(x, t)=\int_{0}^{A} w(x, \alpha) u(x, t, \alpha) d \alpha \text { in } \Omega \times(0, T) .
\end{gathered}
$$

Here $\Phi\left(P_{w}(x, t)\right)$ is the total population with special weight at time $t$ and location $x$ where $A$ is the maximal age of the individual. The weight function $w$ in $P_{w}$ is positive in $L^{\infty}(\Omega \times(0, A))$ and can help with explaining the variety realistic model, as virus, earthquake and storm waves which give effects differently on each ages. So $\Phi\left(P_{w}(x, t)\right)$ can be considered as effects of external environment for population system such as emigration and earthquake and so on. In a biological system, we may apply the model system (1.1)-(1.5) to animal, fish, plant dynamic models. 
Recently, Kang et al.[14] studied an optimal harvesting problem as follows:

$$
\sup _{v \in \mathcal{U}} J(v)=\int_{Q} v(x, t, a) g(x, t, a) u(x, t, a) d x d t d a
$$

subject to the model system (1.1)-(1.5), where $\mathcal{U}$ is the set of controllers defined by

$$
\mathcal{U}=\left\{v \in L^{\infty}(Q): \nu_{1}(x, t, a) \leq v(x, t, a) \leq \nu_{2}(x, t, a) \text { a.e., }(x, t, a) \in Q\right\}
$$

for some $\nu_{1}, \nu_{2} \in L^{\infty}(Q), 0 \leq \nu_{1}(x, t, a) \leq \nu_{2}(x, t, a)$, a.e., in $Q, g$ is a given bounded function and $u$ is a solution of the model system (1.1)-(1.5). The crucial part to these developments is to establish the optimality systems which characterize the optimal control.

In this paper, we study the duality problem of the case that some nonlinear terms in the primal problem can be changed to some linear terms in the dual problem. The purpose is to derive the dual problem corresponding to the control problem $(\mathbf{P})$ and to establish the duality theory which finds some conditions that the primal and dual problems can be connected by the duality relation. Lastly, a theoretical example to illustrate our results is given. The method of proof is based on game theory and the similar method with some inequality techniques given in [13].

\section{Preliminaries}

In place throughout the paper, we need the following hypotheses for the control problem $(\mathbf{P})$ :

(H1) The fertility rate $\beta$ satisfies $\beta \in L^{\infty}(Q), \beta(x, t, a) \geq 0$ a.e. $(x, t, a) \in Q$ and is decreasing.

(H2) The death rate $\mu$ satisfies $\mu \in L^{\infty}\left(\bar{\Omega} \times[0, T] \times[0, A) \times L^{\infty}(Q)\right)$ and $\mu$ is Lipschitz continuous with respect to the variable $u$.

(H3) $\Phi:[0, \infty) \rightarrow[0, \infty)$ is bounded and Lipschitz continuous, i.e., there exist a constant $L>0$ such that

$$
\left|\Phi\left(\psi_{1}\right)-\Phi\left(\psi_{2}\right)\right| \leq L\left|\psi_{1}-\psi_{2}\right|
$$

and $\Phi:[0, \infty) \rightarrow[0, \infty)$ is continuously differentiable.

(H4) $u_{0} \in L^{\infty}(\Omega \times(0, A)), u_{0}(x, a) \geq 0$ a.e., $(x, a) \in \Omega \times(0, A)$.

(H5) $g \in L^{\infty}(Q), g(x, t, a) \geq 0$ a.e., $(x, t, a) \in Q$.

(H6) $w$ is a nonnegative bounded and measurable function in $L^{\infty}(\Omega \times(0, A))$ with $0 \leq w(x, a) \leq 1$ for all $(x, a) \in \Omega \times(0, A)$. 
(H7) $\mu(u) u$ is a concave function of $u$, i.e. $\mu(u) u$ satisfies the following condition

$$
\mu\left(u^{\circ}\right) u^{\circ}-\mu(\bar{u}) \bar{u} \geq\left(\mu_{u}\left(u^{\circ}\right) u^{\circ}+\mu\left(u^{\circ}\right)\right)\left(u^{\circ}-\bar{u}\right),
$$

where $\mu_{u}$ means the derivatives of $\mu$ with respect to $u$.

(H8) $\Phi(\psi(u)) u$ satisfies the following condition

$\Phi\left(\psi\left(u^{\circ}\right)\right) u^{\circ}-\Phi(\psi(\bar{u})) \bar{u} \geq \Phi_{\psi}\left(\psi\left(u^{\circ}\right)\right)\left(\psi\left(u^{\circ}\right)-\psi(\bar{u})\right) u^{\circ}+\Phi\left(\psi\left(u^{\circ}\right)\right)\left(u^{\circ}-\bar{u}\right)$,

where $\Phi_{\psi}$ is the derivatives of $\Phi$ with respect to $\psi$.

Theorem 2.1([14]). Let the hypotheses $(H 1)-(H 6)$ hold. Then there exist a nonnegative solution pair $(u, v)$ and a adjoint solution $p$ to State Equation(SE) and Adjoint Equation(AE), respectively. Also, there exists a solution pair $(u, v, p)$ satisfying the necessary condition for optimality.

- State Equation(SE):

$$
\begin{aligned}
& \frac{\partial u}{\partial t}(x, t, a)+\frac{\partial u}{\partial a}(x, t, a)-k \Delta_{x} u(x, t, a)+\mu(x, t, a, u(x, t, a)) u(x, t, a) \\
& \quad+\Phi\left(P_{w}(x, t)\right) u(x, t, a)=-v(x, t, a) u(x, t, a) \text { in } Q=\Omega \times(0, T) \times(0, A), \\
& \frac{\partial u}{\partial \eta}(x, t, a)=0 \text { on } \Sigma=\partial \Omega \times(0, T) \times(0, A), \\
& u(x, 0, a)=u_{0}(x, a) \text { in } \Omega \times(0, A), \\
& u(x, t, 0)=\int_{0}^{A} \beta(x, t, a) u(x, t, a) d a \text { in } \Omega \times(0, T), \\
& P_{w}(x, t)=\int_{0}^{A} w(x, \alpha) u(x, t, \alpha) d \alpha \text { in } \Omega \times(0, T) .
\end{aligned}
$$

- Adjoint Equation(AE):

$$
\begin{aligned}
& -\frac{\partial p}{\partial t}(x, t, a)-\frac{\partial p}{\partial a}(x, t, a)-k \Delta_{x} p(x, t, a) \\
& +\mu_{u}(x, t, a, u(x, t, a)) u(x, t, a) p(x, t, a) \\
& +\mu(x, t, a, u(x, t, a)) p(x, t, a)+\Phi\left(P_{w}(x, t)\right) p(x, t, a) \\
& +\int_{0}^{A} w(x, a) \Phi_{P_{w}}\left(P_{w}(x, t)\right) u(x, t, \alpha) p(x, t, \alpha) d \alpha-\beta(x, t, a) p(x, t, 0) \\
= & -v(x, t, a) g(x, t, a)-v(x, t, a) p(x, t, a) \text { in } Q \\
= & \Omega \times(0, T) \times(0, A)
\end{aligned}
$$

$$
\frac{\partial p}{\partial \eta}(x, t, a)=0 \text { on } \Sigma=\partial \Omega \times(0, T) \times(0, A),
$$




$$
\begin{aligned}
& p(x, T, a)=p(T)=0 \quad \text { in } \Omega \times(0, A), \\
& p(x, t, A)=p(A)=0 \text { in } \Omega \times(0, T) .
\end{aligned}
$$

Here $\mu_{u}$ is the derivatives of $\mu$ with respect to $u$ and $\Phi_{P_{w}}$ is the derivatives of $\Phi$ with respect to $P_{w}$.

- Necessary condition for optimality : Suppose that $\left(u^{\circ}, v^{\circ}\right)$ is a optimal solution pair for the control problem $(\mathbf{P})$. If $p$ is a solution of the adjoint system (2.1)-(2.4) then we have

$$
v^{\circ}(x, t, a)= \begin{cases}\nu_{1}(x, t, a) & \text { if }(g+p)(x, t, a)<0 \text { and } u^{\circ}(x, t, a) \neq 0, \\ {\left[\nu_{1}(x, t, a), \nu_{2}(x, t, a)\right]} & \text { if }(g+p)(x, t, a)=0 \text { or } u^{\circ}(x, t, a)=0, \\ \nu_{2}(x, t, a) & \text { if }(g+p)(x, t, a)>0 \text { and } u^{\circ}(x, t, a) \neq 0 .\end{cases}
$$

Here $\nu_{1}, \nu_{2}$ are the functions in the controllers set $\mathcal{U}$.

\section{Dual problem}

We associate another control problem dual to the control(primal) problem (P) so called the dual problem. In order to describe it we need a functional $K$ defined by

$K(p(x, t, a), u(x, t, a))=\sup _{v \in \mathcal{U}}\{v(x, t, a) g(x, t, a) u(x, t, a)+p(x, t, a) v(x, t, a) u(x, t, a)\}$.

We call the following control problem the dual problem (D) corresponding to the control problem $(P)$ :

$$
\text { (D) } \quad \begin{aligned}
\inf _{p \in \mathcal{V}} \int_{Q} K(p(x, t, a), u(x, t, a))+p(x, t, a)\left(\frac{\partial u(x, t, a)}{\partial t}+\frac{\partial u(x, t, a)}{\partial a}\right. \\
\left.-k \triangle_{x} u(x, t, a)+\mu(x, t, a, u(x, t, a)) u(x, t, a)+\Phi\left(P_{w}(x, t)\right) u(x, t, a)\right) d x d t d a
\end{aligned}
$$

subject to the adjoint system (2.1)-(2.4). Here $\mathcal{V}$, the set of controllers, is given by

$$
\mathcal{V}=\left\{v \in W^{1, \infty}(Q): p \text { is a positive solution of }(\mathrm{AE})\right\} .
$$

In this section, we establish a duality theorem saying that the primal problem $(\mathbf{P})$ is equal to the dual problem $(\mathbf{D})$. For the proof of the main theorem, we need the following lemma, which is the result of Theorem 1.1 in [11]. 
Lemma 3.1. Assume that the function $v \rightarrow J(v)$ is strictly concave Gateaux differentiable and $v \in \mathcal{U}$. Then the unique element $v^{\circ}$ in $\mathcal{U}$ satisfying $J\left(v^{\circ}\right)=$ $\sup _{v \in \mathcal{U}} J(v)$ is characterized by

$$
J^{\prime}\left(v^{\circ}\right)\left(v-v^{\circ}\right) \leq 0 .
$$

Remark 3.2. The objective functional $J(v)=\int_{Q} v(x, t, a) g(x, t, a) u(x, t, a) d x d t d a$ is strictly concave Gateaux differentiable for $v \in \mathcal{U}$.

Thoerem 3.3. If the assumptions $(H 1)-(H 8)$ hold and $\left(u^{\circ}, v^{\circ}\right)$ attains the supremum of the primal problem $(\mathbf{P})$ then there exists $p^{\circ}$ which is positive adjoint solution of the adjoint system (2.1)-(2.4) such that $\left(p^{\circ}, u^{\circ}\right)$ attains the infimum of the dual problem $(\mathbf{D})$. Furthermore, the supremum of the primal problem $(\mathbf{P})$ is equal to the infimum of the dual problem (D).

Proof. First, we prove the weak duality theorem i.e., the infimum of the dual problem $(\mathbf{D})$ is equal to or greater than the supremum of the primal problem $(\mathbf{P})$. Let $v(\cdot) \in \mathcal{U}$ be any admissible control and fix it for a moment. Due to Theorem 2.1, there exists a solution $u$ of the model system (1.1)-(1.5). Let $\bar{u}$ be a solution of the model system (1.1)-(1.5) corresponding to $v$. To this $v$ we associate the following problem $\left(D_{u}\right)$ :

$$
\begin{aligned}
& \inf _{p \in \mathcal{V}} \int_{Q} v(x, t, a) g(x, t, a) u(x, t, a)+p(x, t, a) v(x, t, a) u(x, t, a) \\
& \quad+p(x, t, a)\left(\frac{\partial u(x, t, a)}{\partial t}+\frac{\partial u(x, t, a)}{\partial a}-k \triangle_{x} u(x, t, a)\right. \\
& \left.\quad+\mu(x, t, a, u(x, t, a)) u(x, t, a)+\Phi\left(P_{w}(x, t)\right) u(x, t, a)\right) d x d t d a .
\end{aligned}
$$

If there is no solution $(p, u)$ satisfying the adjoint system (2.1)-(2.4) then we define the infimum of the problem $\left(D_{u}\right)$ to be $\infty$. If $\left(p^{\circ}, u^{\circ}\right)$ is an arbitrary positive solution of the adjoint system (2.1)-(2.4) then we can show that

$$
\begin{aligned}
& \int_{Q} v(x, t, a) g(x, t, a) u^{\circ}(x, t, a)+p^{\circ}(x, t, a) v(x, t, a) u^{\circ}(x, t, a) \\
& \quad+p^{\circ}(x, t, a)\left(\frac{\partial u^{\circ}(x, t, a)}{\partial t}+\frac{\partial u^{\circ}(x, t, a)}{\partial a}-k \triangle_{x} u^{\circ}(x, t, a)\right. \\
& \left.\quad+\mu\left(x, t, a, u^{\circ}(x, t, a)\right) u^{\circ}(x, t, a)+\Phi\left(P_{w}^{\circ}(x, t)\right) u^{\circ}(x, t, a)\right) d x d t d a \\
& \quad-\int_{Q} v(x, t, a) g(x, t, a) \bar{u}(x, t, a) d x d t d a \geq 0 .
\end{aligned}
$$

To do this, using conditions $(H 7),(H 8)$ and integration by part, we obtain that 


$$
\begin{aligned}
& \int_{Q} v(x, t, a) g(x, t, a) u^{\circ}(x, t, a)+p^{\circ}(x, t, a) v(x, t, a) u^{\circ}(x, t, a) \\
& +p^{\circ}(x, t, a)\left(\frac{\partial u^{\circ}(x, t, a)}{\partial t}+\frac{\partial u^{\circ}(x, t, a)}{\partial a}-k \triangle_{x} u^{\circ}(x, t, a)\right. \\
& \left.+\mu\left(x, t, a, u^{\circ}(x, t, a)\right) u^{\circ}(x, t, a)+\Phi\left(P_{w}^{\circ}(x, t)\right) u^{\circ}(x, t, a)\right) d x d t d a \\
& -\int_{Q} v(x, t, a) g(x, t, a) \bar{u}(x, t, a) d x d t d a \\
& =\int_{Q} v(x, t, a) g(x, t, a) u^{\circ}(x, t, a)-\int_{Q} v(x, t, a) g(x, t, a) \bar{u}(x, t, a) d x d t d a \\
& +\int_{Q} p^{\circ}(x, t, a)\left(v(x, t, a) u^{\circ}(x, t, a)+\frac{\partial u^{\circ}(x, t, a)}{\partial t}+\frac{\partial u^{\circ}(x, t, a)}{\partial a}\right. \\
& \left.-k \triangle_{x} u^{\circ}(x, t, a)+\mu\left(x, t, a, u^{\circ}(x, t, a)\right) u^{\circ}(x, t, a)+\Phi\left(P_{w}^{\circ}(x, t)\right) u^{\circ}(x, t, a)\right) d x d t d a \\
& +\int_{Q} p^{\circ}(x, t, a)\left(-v(x, t, a) \bar{u}(x, t, a)-\frac{\partial \bar{u}(x, t, a)}{\partial t}-\frac{\partial \bar{u}(x, t, a)}{\partial a}\right. \\
& \left.+k \triangle_{x} \bar{u}(x, t, a)-\mu(x, t, a, \bar{u}(x, t, a)) \bar{u}(x, t, a)-\Phi\left(\bar{P}_{w}(x, t)\right) \bar{u}(x, t, a)\right) d x d t d a \\
& =\int_{Q} v(x, t, a) g(x, t, a)\left(u^{\circ}(x, t, a)-\bar{u}(x, t, a)\right) d x d t d a \\
& +\int_{Q} p^{\circ}(x, t, a) v(x, t, a)\left(u^{\circ}(x, t, a)-\bar{u}(x, t, a)\right) d x d t d a \\
& +\int_{Q} p^{\circ}(x, t, a)\left(\frac{\partial u^{\circ}}{\partial t}(x, t, a)-\frac{\partial \bar{u}}{\partial t}(x, t, a)\right) d x d t d a \\
& +p^{\circ}(x, t, a)\left(\frac{\partial u^{\circ}}{\partial a}(x, t, a)-\frac{\partial \bar{u}}{\partial a}(x, t, a)\right) d x d t d a \\
& +p^{\circ}(x, t, a)\left(-k \triangle_{x} u^{\circ}(x, t, a)+k \triangle_{x} \bar{u}(x, t, a)\right) d x d t d a \\
& +\int_{Q} p^{\circ}(x, t, a)\left(\mu\left(x, t, a, u^{\circ}(x, t, a)\right) u^{\circ}(x, t, a)-\mu(x, t, a, \bar{u}(x, t, a)) \bar{u}(x, t, a)\right) d x d t d a \\
& +\int_{Q} p^{\circ}(x, t, a)\left(\Phi\left(P_{w}^{\circ}(x, t)\right) u^{\circ}(x, t, a)-\Phi\left(\bar{P}_{w}(x, t)\right) \bar{u}(x, t, a)\right) d x d t d a \\
& \geq \int_{Q}\left\{v(x, t, a) g(x, t, a)+p^{\circ}(x, t, a) v(x, t, a)-\frac{\partial p^{\circ}}{\partial t}(x, t, a)-\frac{\partial p^{\circ}}{\partial t}(x, t, a)\right. \\
& -p^{\circ}(x, t, 0) \beta(x, t, a)-k \triangle_{x} p^{\circ}(x, t, a)+p^{\circ}(x, t, a) \mu_{u}\left(x, t, a, u^{\circ}(x, t, a)\right) u^{\circ}(x, t, a) \\
& +p^{\circ}(x, t, a) \mu\left(x, t, a, u^{\circ}(x, t, a)\right)+\Phi\left(P_{w}^{\circ}(x, t)\right) p^{\circ}(x, t, a) \\
& \left.+\Phi_{P_{w}}\left(P_{w}^{\circ}(x, t)\right) \int_{0}^{A} w(x, a) p^{\circ}(x, t, \alpha) u^{\circ}(x, t, \alpha) d \alpha\right\}\left(u^{\circ}(x, t, a)-\bar{u}(x, t, a)\right) d Q \\
& =0 \text {. }
\end{aligned}
$$


Let us denote by $d(v)$ the infimum of the problem $\left(D_{u}\right)$. Then the above argument shows that

$$
d(v) \geq J(\bar{u}, v)
$$

holds for every $v \in \mathcal{U}$. If we denote by $L(p, u, v)$ the objective function of the problem $\left(D_{u}\right)$, that is,

$$
\begin{aligned}
L(p, u, v)=\quad \int_{Q} v(x, t, a) g(x, t, a) u(x, t, a)+p(x, t, a) v(x, t, a) u(x, t, a) \\
\quad+p(x, t, a)\left(\frac{\partial u(x, t, a)}{\partial t}+\frac{\partial u(x, t, a)}{\partial a}-k \triangle_{x} u(x, t, a)\right. \\
\left.\quad+\mu(x, t, a, u(x, t, a)) u(x, t, a)+\Phi\left(P_{w}(x, t)\right) u(x, t, a)\right) d x d t d a
\end{aligned}
$$

then it follows from (3.2) and a well-known inequality of game theory that

$$
\inf _{p} \sup _{v} L(p, u, v) \geq \sup _{v} \inf _{p} L(p, u, v)=\sup _{v} d(v) \geq \sup _{\bar{u}, v} J(\bar{u}, v) .
$$

For given $v(\cdot) \in \mathcal{U}$, we have

$$
\begin{aligned}
& \int_{Q} K(p(x, t, a))+p(x, t, a)\left(\frac{\partial u(x, t, a)}{\partial t}+\frac{\partial u(x, t, a)}{\partial a}-k \triangle_{x} u(x, t, a)\right. \\
& \left.\quad+\mu(x, t, a, u(x, t, a)) u(x, t, a)+\Phi\left(P_{w}(x, t)\right) u(x, t, a)\right) d x d t d a \\
& =\sup _{v} L(p, u, v) .
\end{aligned}
$$

If we denote by $\inf (\mathbf{D})$ the infimum of $(\mathbf{D})$ and $\sup (\mathbf{P})$ the supremum of $(\mathbf{P})$, then we obtain that

$$
\inf (\mathbf{D}) \geq \sup (\mathbf{P}) \text {. }
$$

We next prove the duality theorem that under certain conditions $\sup (\mathbf{P})$ coincides with $\inf (\mathbf{D})$. If $\left(u^{\circ}, v^{\circ}\right)$ attains $\sup (\mathbf{P})$, then, by Theorem 2.1 and Lemma 3.1, there exists $p^{\circ}>0$ satisfying

$$
\begin{aligned}
& -\frac{\partial p^{\circ}}{\partial t}(x, t, a)-\frac{\partial p^{\circ}}{\partial a}(x, t, a)-k \Delta_{x} p^{\circ}(x, t, a)+\mu_{u}^{\circ}\left(x, t, a, u^{\circ}(x, t, a)\right) u^{\circ}(x, t, a) p^{\circ}(x, t, a) \\
& \quad+\mu\left(x, t, a, u^{\circ}(x, t, a)\right) p^{\circ}(x, t, a)+\Phi\left(P_{w}^{\circ}(x, t)\right) p^{\circ}(x, t, a) \\
& \quad+\int_{0}^{A} w(x, a) \Phi_{P_{w}}\left(P_{w}^{\circ}(x, t)\right) u^{\circ}(x, t, \alpha) p^{\circ}(x, t, \alpha) d \alpha-\beta(x, t, a) p^{\circ}(x, t, 0) \\
& =-v^{\circ}(x, t, a) g(x, t, a)-v^{\circ}(x, t, a) p^{\circ}(x, t, a) \text { in } Q, \\
& \frac{\partial p^{\circ}}{\partial \eta}(x, t, a)=0 \text { on } \Sigma, \\
& p^{\circ}(x, T, a)=p^{\circ}(T)=0 \text { in } \Omega \times(0, A), \\
& p^{\circ}(x, t, A)=p^{\circ}(A)=0 \text { in } \Omega \times(0, T),
\end{aligned}
$$


and

$$
\int_{Q} v u^{\circ} p^{\circ}+v g u^{\circ} d x d t d a \leq \int_{Q} v^{\circ} u^{\circ} p^{\circ}+v^{\circ} g u^{\circ} d x d t d a .
$$

Moreover, we have

$$
K\left(p^{\circ}(x, t, a)\right)=v^{\circ} g u^{\circ}+p^{\circ} v^{\circ} u^{\circ} \text { a.e., }(x, t, a) \in Q .
$$

Therefore, we conclude that

$$
\begin{aligned}
& \int_{Q} K\left(p^{\circ}(x, t, a)\right)+p^{\circ}(x, t, a)\left(\frac{\partial u^{\circ}(x, t, a)}{\partial t}+\frac{\partial u^{\circ}(x, t, a)}{\partial a}-k \triangle_{x} u^{\circ}(x, t, a)\right. \\
& \left.\quad+\mu\left(x, t, a, u^{\circ}(x, t, a)\right) u^{\circ}(x, t, a)+\Phi\left(P_{w}^{\circ}(x, t)\right) u^{\circ}(x, t, a)\right) d x d t d a \\
& =\int_{Q} v^{\circ}(x, t, a) g(x, t, a) u^{\circ}(x, t, a)+p(x, t, a)\left(\frac{\partial u^{\circ}(x, t, a)}{\partial t}+\frac{\partial u^{\circ}(x, t, a)}{\partial a}\right. \\
& \quad-k \triangle_{x} u^{\circ}(x, t, a)+\mu\left(x, t, a, u^{\circ}(x, t, a)\right) u^{\circ}(x, t, a)+\Phi\left(P_{w}^{\circ}(x, t)\right) u^{\circ}(x, t, a) \\
& \left.\quad+v^{\circ}(x, t, a) u^{\circ}(x, t, a)\right) d x d t d a \\
& =\int_{Q} v^{\circ}(x, t, a) g(x, t, a) u^{\circ}(x, t, a) d x d t d a .
\end{aligned}
$$

By the weak duality theorem, the supremum of the primal problem $(\mathbf{P})$ is equal to the infimum of the dual problem $(\mathbf{D})$ and that $\left(p^{\circ}, u^{\circ}\right)$ attains the infimum of the dual problem $(\mathbf{D})$. This completes the proof.

We now briefly illustrate how our results apply to optimal control problems.

Example 3.4. We consider an optimal control problem as follows:

$$
\left(\mathbf{P}^{\prime}\right) \quad \sup _{v \in \mathcal{U}} J(v)=\int_{Q} v(x, t, a) g(x, t, a) u(x, t, a) d x d t d a
$$

subject to

$$
\begin{aligned}
& \frac{\partial u}{\partial t}(x, t, a)+\frac{\partial u}{\partial a}(x, t, a)-k \Delta_{x} u(x, t, a)+\frac{1}{\sqrt{u(x, t, a)}} u(x, t, a) \\
& +\left(K-\left(\int_{0}^{A} \frac{1}{\sqrt{2 \pi}} e^{-\frac{\alpha^{2}}{2}} u(x, t, \alpha) d \alpha\right)^{2}\right) u(x, t, a)=-v(x, t, a) u(x, t, a) \\
& \text { in } Q=\Omega \times(0, T) \times(0, A), \\
& \frac{\partial u}{\partial \eta}(x, t, a)=0 \text { on } \Sigma=\partial \Omega \times(0, T) \times(0, A), \\
& u(x, 0, a)=u_{0}(x, a) \text { in } \Omega \times(0, A), \\
& u(x, t, 0)=\int_{0}^{A} \beta(x, t, a) u(x, t, a) d a \text { in } \Omega \times(0, T) \\
& P_{w}(x, t)=\int_{0}^{A} \frac{1}{\sqrt{2 \pi}} e^{-\frac{\alpha^{2}}{2}} u(x, t, \alpha) d \alpha \text { in } \Omega \times(0, T) .
\end{aligned}
$$


Here, we take $\mu(u)=1 / \sqrt{u}, w(x, \alpha)=\frac{1}{\sqrt{2 \pi}} e^{-\frac{\alpha^{2}}{2}}, 0 \leq \alpha \leq A$ and

$$
\Phi(\psi)= \begin{cases}K-\psi^{2}, & K>0, \quad 0 \leq \psi \leq \sqrt{K} \\ 0, & \text { otherwise }\end{cases}
$$

We will check hypotheses $(H 7)$ and $(H 8)$.

For $u^{\circ}>\bar{u} \geq 0$, we get

$$
\begin{aligned}
\left(\mu_{u}\left(u^{\circ}\right) u^{\circ}+\mu\left(u^{\circ}\right)\right) & =\left(-\frac{1}{2} \frac{1}{\sqrt{u^{\circ}}}+\frac{1}{\sqrt{u^{\circ}}}\right)=\frac{1}{2} \frac{1}{\sqrt{u^{\circ}}} \\
& \leq \frac{1}{\sqrt{u^{\circ}}+\sqrt{\bar{u}}} \\
& =\frac{\sqrt{u^{\circ}}-\sqrt{\bar{u}}}{u^{\circ}-\bar{u}}=\frac{\mu\left(u^{\circ}\right) u^{\circ}-\mu(\bar{u}) \bar{u}}{u^{\circ}-\bar{u}} .
\end{aligned}
$$

Since $P_{w}^{\circ}=\int_{0}^{A} \frac{1}{\sqrt{2 \pi}} e^{-\frac{\alpha^{2}}{2}} u^{\circ} d \alpha \geq \bar{P}_{w}=\int_{0}^{A} \frac{1}{\sqrt{2 \pi}} e^{-\frac{\alpha^{2}}{2}} \bar{u} d \alpha$ for $u^{\circ} \geq \bar{u}$, we obtain

$$
\begin{aligned}
& \Phi\left(P_{w}^{\circ}\right) u^{\circ}-\Phi\left(\bar{P}_{w}\right) \bar{u} \\
= & \Phi\left(P_{w}^{\circ}\right) u^{\circ}-\Phi\left(\bar{P}_{w}\right) u^{\circ}+\Phi\left(\bar{P}_{w}\right) u^{\circ}-\Phi\left(\bar{P}_{w}\right) \bar{u} \\
= & \left(K-\left(P_{w}^{\circ}\right)^{2}\right) u^{\circ}-\left(K-\left(\bar{P}_{w}\right)^{2}\right) u^{\circ}+\left(K-\left(\bar{P}_{w}\right)^{2}\right) u^{\circ}-\left(K-\left(\bar{P}_{w}\right)^{2}\right) \bar{u} \\
\geq & -2 P_{w}^{\circ}\left(P_{w}^{\circ}-\bar{P}_{w}\right) u^{\circ}+\left(K-\left(P_{w}^{\circ}\right)^{2}\right)\left(u^{\circ}-\bar{u}\right) \\
= & \Phi_{P_{w}}\left(P_{w}^{\circ}\right)\left(P_{w}^{\circ}-\bar{P}_{w}\right) u^{\circ}+\Phi\left(P_{w}^{\circ}\right)\left(u^{\circ}-\bar{u}\right) .
\end{aligned}
$$

The dual problem to $\left(\mathbf{P}^{\prime}\right)$ can be formulated via the dual problem of this abstract one. In order to obtain it, we need to evaluate $K(p(x, t, a), u(x, t, a))$ which given by

$$
K(p(x, t, a))=\sup _{v \in \mathcal{U}}\{v(x, t, a) g(x, t, a) u(x, t, a)+p(x, t, a) v(x, t, a) u(x, t, a)\} .
$$

Hence the dual problem to $\left(\mathbf{P}^{\prime}\right)$ becomes

$$
\begin{aligned}
\left(\mathbf{D}^{\prime}\right) & \inf _{p \in \mathcal{V}} \int_{Q} K(p(x, t, a))+p(x, t, a)\left(\frac{\partial u(x, t, a)}{\partial t}+\frac{\partial u(x, t, a)}{\partial a}-k \triangle_{x} u(x, t, a)\right. \\
& \left.+\frac{1}{\sqrt{u(x, t, a)}} u(x, t, a)+\left(K-\left(\int_{0}^{A} \frac{1}{\sqrt{2 \pi}} e^{-\frac{\alpha^{2}}{2}} u(x, t, \alpha) d \alpha\right)^{2}\right) u(x, t, a)\right) d x d t d a
\end{aligned}
$$


subject to

$$
\begin{aligned}
& -\frac{\partial p}{\partial t}(x, t, a)-\frac{\partial p}{\partial a}(x, t, a)-k \Delta_{x} p(x, t, a)-\frac{1}{2} \frac{1}{\sqrt{u(x, t, a)}} p(x, t, a) \\
& +\frac{1}{\sqrt{u(x, t, a)}} p(x, t, a)+\left(K-\left(\int_{0}^{A} \frac{1}{\sqrt{2 \pi}} e^{-\frac{\alpha^{2}}{2}} u(x, t, \alpha) d \alpha\right)^{2}\right) p(x, t, a) \\
& +\int_{0}^{A} \frac{1}{\sqrt{2 \pi}} e^{-\frac{a^{2}}{2}}\left(-2 \int_{0}^{A} \frac{1}{\sqrt{2 \pi}} e^{-\frac{\alpha^{2}}{2}} u(x, t, \alpha) d \alpha\right) u(x, t, \alpha) p(x, t, \alpha) d \alpha \\
& -\beta(x, t, a) p(x, t, 0) \\
& =-v(x, t, a) g(x, t, a)-v(x, t, a) p(x, t, a) \text { in } Q,
\end{aligned}
$$

$$
\begin{gathered}
\frac{\partial p}{\partial \eta}(x, t, a)=0 \text { on } \Sigma, \\
p(x, T, a)=p(T)=0 \text { in } \Omega \times(0, A), \\
p(x, t, A)=p(A)=0 \text { in } \Omega \times(0, T) .
\end{gathered}
$$

From the above facts and Theorem 3.3, we obtain the following result:

Theorem 3.5. If the assumptions $(H 1)-(H 8)$ are satisfied and $\left(u^{\circ}, v^{\circ}\right)$ attains the supremum of $\left(\mathbf{P}^{\prime}\right)$ then there exists $p^{\circ}$ which is a positive adjoint solution of the adjoint system (4.1)-(4.4) such that $\left(p^{\circ}, u^{\circ}\right)$ attains the infimum of $\left(\mathbf{D}^{\prime}\right)$. Furthermore, the supremum of $\left(\mathbf{P}^{\prime}\right)$ is equal to the infimum of $\left(\mathbf{D}^{\prime}\right)$.

\section{References}

[1] B. Ainseba, S. Anita and M. Langlais, Optimal control for a nonlinear agestructured population dynamics model, Electronic Journal of Differential Equations, 2002(28)(2002), 1-9.

[2] S. Anita, Optimal harvesting for a nonlinear age-dependent population dynamics, Journal of Mathematical Analysis and Applications, 226(1998), 6-22.

[3] Tadeusz Antczak, Optimal conditions and duality for nondifferentiable multiobjective programming problems involving d-r-type I functions, Journal of Computational and Applied Mathematics, 225(2009), 236-250.

[4] V. Barbu and M. Lannelli, Optimal control of population dynamics, Journal of Optimization Theory and Applications, 102(1)(1999), 1-14. 
[5] F. Brauer and C. Castillo-Chávez, Mathematical models in population biology and epidemiology, With 117 Illustrations, 2001 Springer-Verlag New York, Inc..

[6] A. V. Bulatov and V. F. Krotov, On dual problems of optimal control, Automation and Remote control, 69(10)(2008), 1653-1662.

[7] W. L. Chan, Duality in the optimal control of non-well-posed parabolic equations with positive control, Journal of Mathematical Analysis and Applications, 107(1985), 509519 .

[8] Nobuyuki Kato, Optimal harvesting for nonlinear size-structured population dynamics, Journal of Mathematical Analysis and Applications, 342(2008), 1388-1398.

[9] K. R. Fister and S. Lenhart, Optimal control of a competitive system with agestructure, Journal of Mathematical Analysis and Applications, 291(2004), 526-537.

[10] K. R. Fister and S. Lenhart, Optimal harvesting in an age-structured predator-prey model, Applied Mathematics and Optimization, 54(2006), 1-15.

[11] J. L. Lions, Optimal control of systems governed by partial differential equations, Springer-Verlag Berlin Heidelberg New York 1971.

[12] J. Y. Park and M. J. Lee, Duality in the optimal control problems for hyperbolic distributed parameter systems with damping terms, Journal of Mathematical Analysis and Applications, 227(1998), 449-461.

[13] J. Y. Park and M. J. Lee, Duality in the optimal control of distributed parameter systems governed by hyperbolic equations, Indian J. Pure Appl. Math., 31(4)(2000), 451-460.

[14] Y. H. Kang, J. B. Lee, M. J. Lee, Yongkuk Kim and I. H. Jung, Optimal harvesting for an age-spatial structured population model with an external environment, submitted for publication.

[15] S. Tanimoto, Duality in the optimal control of non-well-posed distributed systems, Journal of Mathematical Analysis and Applications, 171(1992), 277-287.

[16] G. Feichtinger, G. Tragler and V. M. Veliov, Optimal conditions for age-structured control systems, Journal of Mathematical Analysis and Applications, 95(1996), 25-42.

[17] Y. Y. Zhou and X. Q. Yang, Duality and penalization in optimization via an augmented lagrangian function with applications, Journal of Optimization Theory and Applications, 140(2009), 171-188. 